Frank Schweitzer, Benno Tilch, Werner Ebeling:

Uphill Motion of Active Brownian Particles in Piecewise Linear Potentials

European Journal of Physics B (1999, in press)

\title{
Uphill Motion of Active Brownian Particles in Piecewise Linear Potentials
}

\author{
Frank Schweitzer ${ }^{1 \star}$, Benno Tilch ${ }^{2}$, Werner Ebeling ${ }^{1}$ \\ 1 Institute of Physics, Humboldt University Berlin, Invalidenstraße 110, D-10115 \\ Berlin, Germany \\ 2 II. Institute of Theoretical Physics, University of Stuttgart, Pfaffenwaldring 57/III, \\ D-70550 Stuttgart, Germany \\ * Present address: GMD Institute for Autonomous intelligent Systems, Schloss Bir- \\ linghoven, 53754 Sankt Augustin, Germany,e-mail: schweitzer@gmd.de
}

\begin{abstract}
We consider Brownian particles with the ability to take up energy from the environment, to store it in an internal depot, and to convert internal energy into kinetic energy of motion. Provided a supercritical supply of energy, these particles are able to move in a "high velocity" or active mode, which allows them to move also against the gradient of an external potential. We investigate the critical energetic conditions of this self-driven motion for the case of a linear potential and a ratchet potential. In the latter case, we are able to find two different critical conversion rates for the internal energy, which describe the onset of a directed net current into the two different directions. The results of computer simulations are confirmed by analytical expressions for the critical parameters and the average velocity of the net current. Further, we investigate the influence of the asymmetry of the ratchet potential on the net current and estimate a critical value for the asymmetry in order to obtain a positive or negative net current.
\end{abstract}

\section{PACS numbers:}

05.40.Jc Brownian motion

05.45.-a Nonlinear dynamics and nonlinear dynamical systems

05.60.-k Transport processes

82.20.Mj Nonequilibrium kinetics

87.10.+e General theory and mathematical aspects 


\section{Introduction}

The motion of a "simple" Brownian particle is due to fluctuations of the surrounding medium, i.e. the result of random impacts of the molecules or atoms of the liquid or gas, the particle is immersed in. This type of motion would be rather considered as passive motion, simply because the Brownian particle does not play an active part in this motion. On the other hand, already in physico-chemical systems a self-driven motion of particles can be found [1]. For instance, small solid particles floating on a liquid surface may produce a chemical substance at a spatially inhomogeneous rate, which locally changes the surface tension. This way the substance induces a net capillary force acting on the particle, which results in the particle's motion.

Recent investigations on interacting self-driven particles show a broad variety of interesting phenomena, such as phase transitions and the emergence of self-ordered motion [2-4]. Here, the focus is on collective effects rather than on the origin of the particle's velocity; i.e. it is usually postulated that the particles move with a certain non-zero velocity.

In this paper, we focus on the energetic aspects of self-driven motion in order to derive conditions for an active mode of motion. Active motion, as the name suggests, occurs under energy consumption, it is related to processes of energy storage and conversion into kinetic energy. For instance on the biological level, cells or simple microorganisms are capable of active, self-driven motion, which in many cases has been successfully described by stochastic equations $[2,5-9]$.

In order to describe both the random aspects and the energetic aspects of active motion, we have introduced a model of active Brownian particles [10-15]. These are Brownian particles with the ability to take up energy from the environment, to store it in an internal depot and to convert internal energy to perform different activities, such as metabolism, motion, change of the environment, or signal-response behavior. Since the focus in this paper is on the energetic aspects of active Brownian particles in a specific potential, possible changes of the environment are neglected here.

A very simple mechanism to take-up the additional energy required for active motion, is the pumping of energy by space-dependent friction [11]. In this case, the friction coefficient $\gamma_{0}$ becomes a space-dependent function, $\gamma(\boldsymbol{r})$, which in a certain spatial range can be also negative. Inside this area the Brownian particle, instead of loosing energy because of dissipative processes, is pumped with energy, which in turn increases its velocity. Provided a supercritical supply of energy, the particle should be able to move with a velocity larger than the average thermal velocity.

While such an approach will be able to model the spatially inhomogeneous supply of energy, it has the drawback not to consider processes of storage and conversion of energy. In fact, with only a space-dependent friction, the Brownian particle is instantaneously accelerated or slowed down, whereas e.g. biological systems have the capability to stretch their supply of energy over a certain time interval. 
Frank Schweitzer, Benno Tilch, Werner Ebeling:

Uphill Motion of Active Brownian Particles in Piecewise Linear Potentials

European Journal of Physics B (1999, in press)

In order to develop a more realistic model of active motion, we have considered an internal energy depot for the Brownian particles [14,15], which allows to store the taken-up energy in the internal depot, from where it can be converted e.g. into kinetic energy, namely for the acceleration of motion. Additionally, the internal dissipation of energy, due to storage and conversion (or metabolism in a biological context) can be considered.

With these extensions, the Brownian particle becomes in fact a Brownian motor [16-18], which is fueled somewhere and then uses the stored energy with a certain efficiency [15] to move forward, also against external forces. Provided a supercritical supply of energy, we have found that the motion of active Brownian particles in the two-dimensional space can become rather complex [14].

In this paper we investigate the one-dimensional motion of an ensemble of Brownian particles with internal energy depot in piecewise linear potentials. One particular example is the ratchet potential, i.e. a periodic potential which lacks the reflection symmetry. Ratchet systems recently attracted much interest with respect to transport phenomena on the microscale, since they provide a mechanism to transfer the undirected motion of Brownian particles into a directed motion. Hence, the term Brownian rectifiers [19] has been established. In order to reveal the microscopic mechanisms resulting in directed movement, different physical ratchet models have been proposed [20], such as forced thermal ratchets [21], or stochastic ratchets [22,23], or fluctuating ratchets [24,25].

The model discussed in this paper, aims to add a new perspective to this problem which is based on the idea of internal energy storage. In Sect. 2, we discuss the basic features of our model without specifying an external potential, and derive the equations of motion both for the general case and the overdamped limit. In particular, we point to the possible existence of a "high velocity" or active mode of motion for the Brownian particle, in addition to the usual passive mode of motion. In Sect. 3 , the necessary conditions for such an active mode of motion are investigated for the case of a linear potential. For the overdamped limit, we derive critical parameters of energy conversion, which allows the particle to move also against the direction of the external force, i.e. to move "uphill" the potential gradient. The results are applied to the motion of Brownian particles in a ratchet potential in Sect. 4. After investigating the deterministic motion of a single particle in Sect. 4.1, we discuss the energetic conditions for the establishment of a net current for the determinsitic motion of an ensemble of particles in Sect. 4.2. We show that, dependent on the conversion of internal into kinetic energy, a net current into different directions can be established. The critical parameters for the energy conversion and the resulting velocity of the net current, which are found by means of computer simulations, are compared with the analytical results obtained in Sect. 2. We also investigate the influence of the asymmetry of the ratchet potential on the establishment of the net current. 


\section{Equations of Motion of Pumped Brownian Dynamics}

The motion of simple Brownian particles in a space-dependent potential, $U(\boldsymbol{r})$ can be described by the Langevin equation:

$$
\dot{\boldsymbol{r}}=\boldsymbol{v} ; m \dot{\boldsymbol{v}}=-\gamma_{0} \boldsymbol{v}-\nabla U(\boldsymbol{r})+\mathcal{F}(t)
$$

where $\gamma_{0}$ is the friction coefficient of the particle at position $\boldsymbol{r}$, moving with velocity $\boldsymbol{v} . \mathcal{F}(t)$ is a stochastic force with strength $D$ and a $\delta$-correlated time dependence

$$
\langle\mathcal{F}(t)\rangle=0 ;\left\langle\mathcal{F}(t) \mathcal{F}\left(t^{\prime}\right)\right\rangle=2 D \delta\left(t-t^{\prime}\right)
$$

Using the fluctuation-dissipation theorem, we assume that the loss of energy resulting from friction, and the gain of energy resulting from the stochastic force, are compensated in the average, and $D$ can be expressed as:

$$
D=k_{B} T \gamma_{0}
$$

where $T$ is the temperature and $k_{B}$ is the Boltzmann constant.

In addition to the dynamics described above, the Brownian particles considered here are pumped with energy from the environment, which they can store in an internal depot. Further, internal energy can be converted into kinetic energy. Considering also internal dissipation, the resulting balance equation for the internal energy depot, $e$, of a pumped Brownian particle is given by:

$$
\frac{d}{d t} e(t)=q(\boldsymbol{r})-c e(t)-d(\boldsymbol{v}) e(t)
$$

$q(\boldsymbol{r})$ is the space-dependent pump rate of energy and $c$ describes the internal dissipation assumed to be proportional to the depot energy. $d(\boldsymbol{v})$ is the rate of conversion of internal into kinetic energy which should be a function of the actual velocity of the particle. A simple ansatz for $d(\boldsymbol{v})$ reads:

$$
d(\boldsymbol{v})=d_{2} v^{2}
$$

where $d_{2}>0$ is the conversion rate of internal into kinetic energy. The energy conversion results in an additional acceleration of the Brownian particle in the direction of movement, expressed by the vector $\boldsymbol{e}_{v}=\boldsymbol{v} / v$. Hence, the equation of motion for the pumped Brownian particles has to consider an additional driving force, $d_{2} e(t) \boldsymbol{v}$. In $[14,15]$, we have postulated a stochastic equation, which is consistent with the Langevin Eq. (1):

$$
\dot{\boldsymbol{r}}=\boldsymbol{v} ; m \dot{\boldsymbol{v}}+\gamma_{0} \boldsymbol{v}+\nabla U(\boldsymbol{r})=d_{2} e(t) \boldsymbol{v}+\mathcal{F}(t)
$$


If we restrict ourselves to the one-dimensional case, i.e. the space coordinate is given by $\boldsymbol{x}$ and further assume $m=1$ for the mass and $q(\boldsymbol{r})=q_{0}=$ const. for influx of energy, the dynamics for the pumped Brownian motion, eqs. (6), (4) can be specified as follows:

$$
\begin{aligned}
\dot{\boldsymbol{x}} & =\boldsymbol{v} \\
\dot{\boldsymbol{v}} & =-\left(\gamma_{0}-d_{2} e(t)\right) \boldsymbol{v}-\frac{\partial U(x)}{\partial x}+\sqrt{2 D} \boldsymbol{\xi}(t) \\
\dot{e} & =q_{0}-c e-d_{2} v^{2} e
\end{aligned}
$$

where $\boldsymbol{\xi}$ is a stochastic force with white-noise fluctuations:

$$
\left\langle\boldsymbol{\xi}(t) \boldsymbol{\xi}\left(t^{\prime}\right)\right\rangle=\delta\left(t-t^{\prime}\right)
$$

In order to find a solution for the coupled Eqs. (7), let us now consider a relaxation of the dynamics on different time scales. If we assume that the velocity $\boldsymbol{v}(t)$ is changing much faster than the space coordinate $\boldsymbol{x}(t)$, for $\boldsymbol{v}(t)$, Eq. (7), a formal solution can be given:

$$
\begin{aligned}
\boldsymbol{v}(t)= & \boldsymbol{v}(0) \exp \left\{-\gamma_{0} t+d_{2} \int_{0}^{t} e\left(t^{\prime}\right) d t^{\prime}\right\} \\
& +\exp \left\{-\gamma_{0} t+d_{2} \int_{0}^{t} e\left(t^{\prime}\right) d t^{\prime}\right\} \\
& \times \int_{0}^{t} \exp \left\{\gamma_{0} t^{\prime}-d_{2} \int_{0}^{t^{\prime}} e\left(t^{\prime \prime}\right) d t^{\prime \prime}\right\} \\
& \times\left[-\nabla U+\sqrt{2 k_{B} T \gamma_{0}} \boldsymbol{\xi}\left(t^{\prime}\right)\right] d t^{\prime}
\end{aligned}
$$

This solution however depends on the integrals over $e(t)$, reflecting the influence of the energy depot on the velocity. If we further assume a fast relaxation of the depot, $e(t)$, compared to the relaxation of the velocity $\boldsymbol{v}(t)$, the corresponding equation of Eq. (7) can be solved, and we obtain with the initial condition $e(0)=0$ the following quasistationary value for the energy depot:

$$
e_{0}=\frac{q_{0}}{c+d_{2} \boldsymbol{v}^{2}}
$$

which yields a possible maximum value of $e_{0}^{\max }=q_{0} / c$.

The overdamped limit is obtained by considering a fast relaxation of the velocities, in which case the set of Eq. (7) can be further reduced to:

$$
\boldsymbol{v}(t)=-\frac{1}{\gamma_{0}-d_{2} e_{0}} \frac{\partial U}{\partial \boldsymbol{x}}+\frac{\sqrt{2 k_{B} T \gamma_{0}}}{\gamma_{0}-d_{2} e_{0}} \boldsymbol{\xi}(t)
$$

We note that, due to the dependence of $e_{0}$ on $\boldsymbol{v}^{2}=\dot{\boldsymbol{x}}^{2}$, Eq. (11) is coupled to Eq. (10). Thus, the overdamped Eq. (11) could be also written in the form:

$$
\left(\gamma_{0}-d_{2} \frac{q_{0}}{c+d_{2} \dot{x}^{2}}\right) \dot{\boldsymbol{x}}=-\frac{\partial U}{\partial \boldsymbol{x}}+\sqrt{2 k_{B} T \gamma_{0}} \boldsymbol{\xi}(t)
$$


Eq. (12) indicates a cubic equation for the velocities in the overdamped limit, i.e. the possible existence of non-trivial solutions for the stationary velocity. For the further discussion, we neglect the stochastic term in Eq. (12) and denote the stationary values of $\boldsymbol{v}(t)$ by $\boldsymbol{v}_{0}(\boldsymbol{x})$. Further, the force resulting from the gradient of the potential, $\boldsymbol{F}(\boldsymbol{x})=-\nabla U$, is introduced. Then, Eq. (12) can be rewritten as:

$$
\left[d_{2} \gamma_{0} v_{0}^{2}-d_{2} \boldsymbol{F} \boldsymbol{v}_{0}-\left(q_{0} d_{2}-c \gamma_{0}\right)\right] \boldsymbol{v}_{0}=c \boldsymbol{F} .
$$

Depending on the value of $\boldsymbol{F}$ and in particular on the sign of the term $\left(q_{0} d_{2}-c \gamma\right)$, Eq. (13) has either one or three real solutions for the stationary velocity, $\boldsymbol{v}_{0}$. The always existing solution expresses a direct response to the force in the form:

$$
\boldsymbol{v}_{0}(\boldsymbol{x}) \sim \boldsymbol{F}(\boldsymbol{x})
$$

This solution results from the analytic continuation of Stokes' law, $\boldsymbol{v}_{0}=\boldsymbol{F} / \gamma_{0}$, which is valid for $d_{2}=0$. We will denote this solution as the "normal response" mode of motion, since the velocity $\boldsymbol{v}$ has the same direction as the force $\boldsymbol{F}$ resulting from the external potential $U(\boldsymbol{x})$.

As long as the supply of the energy depot is small, we will also name the normal mode as the passive mode, because the particle is simply driven by the external force. More interesting is the case of three stationary velocities, $\boldsymbol{v}_{0}$, which significantly depends on the (supercritical) influence of the energy depot. In this case which will be discussed in detail in the following section, the particle will be able to move in a "high velocity" or active mode of motion. For the one-dimensional motion, in the active mode only two different directions are possible, i.e. a motion into or against the direction of the force $\boldsymbol{F}$.

But already in the two-dimensional case there are infinitely different possibilities. This conclusion is of importance when discussing stochastic influences as will be done in a forthcoming paper [26]. In the one-dimensional case, the influence of noise is rather weak, because of the limited number of possible directions, but in the two- and three-dimensional case, the active motion of the particles is very sensitive to stochastic influences, which may determine the direction of motion in the active mode.

\section{Deterministic Motion in a Linear Potential}

\subsection{Stationary Solutions}

In the following, we restrict the discussion to the one-dimensional, deterministic motion of the particle, corresponding to $D=0$ in Eq. (7). Further, we may assume that the force resulting from the gradient of the potential is constant. Then, we have the two coupled equations for $\boldsymbol{v}(t)$ and 
$e(t)$ :

$$
\begin{aligned}
\dot{\boldsymbol{v}} & =-\left(\gamma_{0}-d_{2} e(t)\right) \boldsymbol{v}+\boldsymbol{F} \\
\dot{e} & =q_{0}-c e-d_{2} \boldsymbol{v}^{2} e
\end{aligned}
$$

The stationary solutions of Eq. (15) are obtained from $\dot{\boldsymbol{v}}=0$ and $\dot{e}=0$ :

$$
\boldsymbol{v}_{0}=\frac{\boldsymbol{F}}{\gamma_{0}-d_{2} e_{0}} \quad ; \quad e_{0}=\frac{q_{0}}{c+d_{2} \boldsymbol{v}_{0}^{2}}
$$

which lead to the known cubic polynom for the amount of the constant velocity $v_{0}$, Eq. (13):

$$
d_{2} \gamma_{0} \boldsymbol{v}_{0}^{3}-d_{2} \boldsymbol{F} v_{0}^{2}-\left(q_{0} d_{2}-c \gamma_{0}\right) \boldsymbol{v}_{0}-c \boldsymbol{F}=0
$$

Here $\boldsymbol{v}_{0}^{n+1}$ is defined as a vector $\left|v_{0}\right|^{n} \boldsymbol{v}_{0}$.

For a first insight into the possible solutions of Eq. (17), we consider the force free motion, $\boldsymbol{F}=0$. Then Eq. (17) has either one or three stationary solutions which read:

$$
v_{0}^{(1)}=0, \quad v_{0}^{(2,3)}= \pm \sqrt{\frac{q_{0}}{\gamma_{0}}-\frac{c}{d_{2}}}
$$

The corresponding bifurcation diagram is shown in Fig. 1a which displays a typical fork bifurcation. The bifurcation point is given by:

$$
d_{2}^{b i f}=\frac{c \gamma_{0}}{q_{0}}
$$

Above a critical supply of energy, which is expressed in terms of the conversion parameter $d_{2}$, we find the occurence of two new solutions for the stationary velocity, corresponding to the active modes of motion. For $\boldsymbol{F}=0, v_{0}^{(2)}$ and $v_{0}^{(3)}$ both have the same amount but different directions. Below the critical threshold the passive mode of motion, $v_{0}^{(1)}=0$, is the only stable solution. At the bifurcation point, a change of the stability occurs, consequently above the critical value of $d_{2}$ the active modes of motion are assumed stable.

For the case $\boldsymbol{F}=$ const. $\neq 0$, the analysis of Eq. (17) leads to a different bifurcation diagram shown in Fig. 1b. Above a critical supply of energy, we find the appearance of two high velocity or active modes of motion. One of these active modes has the same direction as the driving force, thus it can be understood as the continuation of the normal solution. As Fig. 1b shows, the former passive normal mode, which holds for subcritical energetic conditions, is transformed into an active normal mode, where the particle moves into the same direction, but with a much higher velocity. Additionally, in the active mode a new high-velocity motion against the direction of the force $\boldsymbol{F}$ becomes possible. While the first active mode would be rather considered as a normal response to the force $\boldsymbol{F}$, the second active mode appears as unnormal (or non-trivial) response, which corresponds to an "uphill" motion (cf. Fig. 2). 

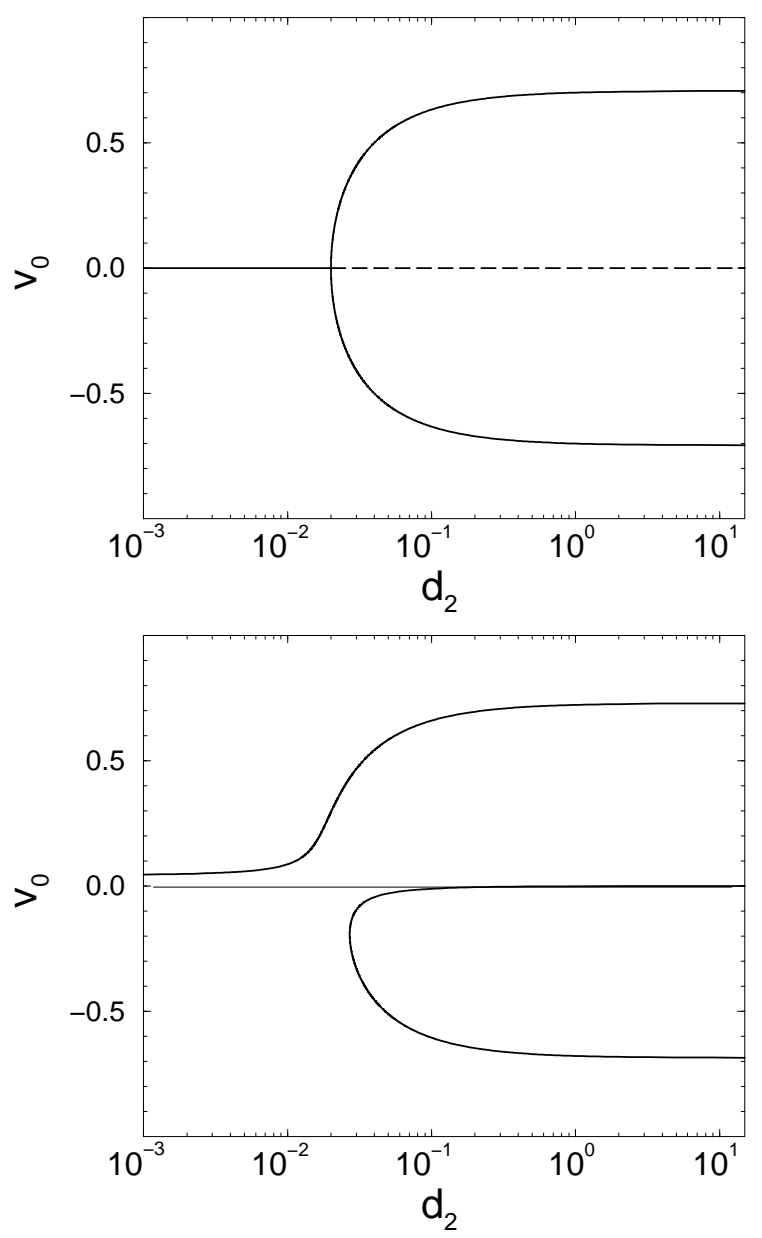

Figure 1: Stationary velocities $v_{0}$, Eq. (17), vs. conversion rate $d_{2}$ : (a: top) for $\boldsymbol{F}=0$, (b: bottom) for $\boldsymbol{F}=+7 / 8$. Above a critical value of $d_{2}$, a negative stationary velocity indicates the posssibility to move against the direction of the force. Parameters: $q_{0}=10, \gamma_{0}=20, c=0.01$.

It is obvious that the particle's motion "downhill" is stable, but the same does not necessarily apply for the possible solution of an "uphill" motion. Thus, in addition to Eq. (17) which provides the values of the stationary solutions, we need a second condition which guarantees the stability of these solutions. Before this is carried out, we want to derive a handy expression for the stationary velocities in the case $\boldsymbol{F} \neq 0$. With the assumption that the term $c \boldsymbol{F}$ is small, the stationary solutions of Eq. (17) can be given as:

$$
v_{0}^{(1)}=0, \quad v_{0}^{(2,3)}=\frac{F}{2 \gamma_{0}} \pm \sqrt{\frac{F^{2}}{4 \gamma_{0}^{2}}+\left(\frac{q_{0}}{\gamma_{0}}-\frac{c}{d_{2}}\right)}
$$

We note that Eq. (20) is a sufficient approximation for the stationary velocities, especially in a 
certain distance from the bifurcation point, thus it will be also used in Sect. 4.1.

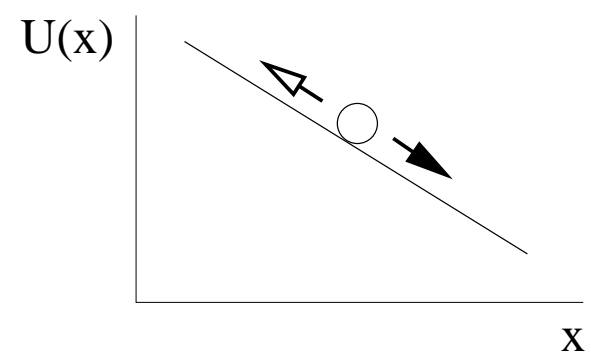

Figure 2: Sketch of the one-dimensional deterministic motion of the particle in the presence of a constant force $\boldsymbol{F}=-\nabla U(x)=$ const.. Provided a supercritical amount of energy from the depot, the particle might be able to move "uphill", i.e. against the direction of the force.

\subsection{Stability Analysis}

For the stability analysis, we consider small fluctuations around the stationary values, $v_{0}$ and $e_{0}$ :

$$
v=v_{0}+\delta v ; \quad e=e_{0}+\delta e ;\left|\frac{\delta v}{v_{0}}\right| \sim\left|\frac{\delta e}{e_{0}}\right| \ll 1
$$

Inserting Eq. (21) into Eq. (15), we find after linearization:

$$
\begin{aligned}
& \dot{\delta v}=\delta v\left(-\gamma_{0}+d_{2} e_{0}\right)+\delta e\left(d_{2} v_{0}\right) \\
& \dot{\delta e}=\delta v\left(-2 d_{2} e_{0} v_{0}\right)+\delta e\left(-c-d_{2} v_{0}^{2}\right)
\end{aligned}
$$

With the ansatz:

$$
\delta v \sim \exp \{\lambda t\} ; \delta e \sim \exp \{\lambda t\}
$$

we find from Eq. (22) the following relation for $\lambda$ :

$$
\begin{aligned}
\lambda^{(1,2)}= & -\frac{1}{2}\left(\gamma_{0}+c+d_{2} v_{0}^{2}-d_{2} e_{0}\right) \\
\pm & \frac{\sqrt{\frac{1}{4}\left(\gamma_{0}+c+d_{2} v_{0}^{2}-d_{2} e_{0}\right)^{2}-\cdots}}{\cdots-c\left(\gamma_{0}-d_{2} e_{0}\right)-d_{2} v_{0}^{2}\left(\gamma_{0}+d_{2} e_{0}\right)}
\end{aligned}
$$

In general, we need to discuss Eq. (24) for the three possible solutions $v_{0}$ which result from Eq. (17). Dependent on whether the $\lambda$ for each solution have real or complex positive or negative values, we are able to classify the types of the possible stationary solutions in this case. The results are 
Frank Schweitzer, Benno Tilch, Werner Ebeling:

Uphill Motion of Active Brownian Particles in Piecewise Linear Potentials

European Journal of Physics B (1999, in press)

\begin{tabular}{|c|c|c|c|c|}
\hline 0 & $<d_{2}<$ & 0.027 & $:$ & 1 stable node \\
\hline 0.027 & $<d_{2}<$ & 0.046 & $:$ & $\begin{array}{l}1 \text { stable node, } 1 \text { instable } \\
\text { node, } 1 \text { saddle point }\end{array}$ \\
\hline 0.046 & $<d_{2}<$ & 2.720 & $:$ & $\begin{array}{l}1 \text { stable focal point, } 1 \\
\text { instable focal point, } 1 \\
\text { saddle point }\end{array}$ \\
\hline 2.720 & $<d_{2}$ & & $:$ & $\begin{array}{l}2 \text { stable focal points, } 1 \\
\text { saddle point }\end{array}$ \\
\hline
\end{tabular}

Table 1: Results for the stability analysis, Eq. (24).

summarized in Table 1. The phase plots shown in Fig. 3a,b present more details. Further, Fig. 4 shows the real part $\Re(\lambda)$ of Eq. (24) for the active mode corresponding to the "uphill" motion of the particle, which is the most interesting one.

We find that below the bifurcation point which is $d_{2}^{b i f}=0.027$ for the given set of parameters, only one stable node exists in the $\{v, e\}$ phase space, which corresponds to the passive normal mode. Then, a subcritical bifurcation occurs which leads to 3 stationary solutions: a stable and an instable node, and a saddle point, since all the $\lambda$ are real. At $d_{2}=0.046$, however, the nodes turn into focal points. With respect to the "uphill motion" we find in Fig. 4 the occurence of an instable node at $d_{2}=0.027$, which then becomes an instable focus for $0.046<d_{2}<2.720$. The respective real parts of $\lambda$ are equal in this range, i.e. the $\lambda^{1,2}$ are complex. The stability condition is satisfied only if $\Re(\lambda) \leq 0$, which is above a second critical value $d_{2}^{\text {crit }}=2.72$ for the given set of parameters. That means, for $d_{2}>2.72$, the instable focal point becomes a stable focus, which is also clearly shown in the phase plots of Fig. 3a,b. In both Figures, we see a stable focal point for positive values of the velocity, $v$, which correspond to the stable motion "downhill", i.e. in the direction of the driving force. For $d_{2}=2.0<d_{2}^{\text {crit }}$, the phase plot for negative values of $v$ shows an instable focal point, which turns into a stable focal point for $d_{2}=4.0>d_{2}^{\text {crit }}$.

Thus, we can conclude that for $d_{2}^{\text {bif }}<d_{2}$ an active mode of motion becomes possible, which also implies the possibility of an "uphill" motion of the particle. However, only for values $d_{2}^{\text {crit }}<d_{2}$, we can expect a stable motion against the direction of the force. The interesting result of a possible stable "uphill-motion" of particles with internal energy depot will be employed in the following section, where we turn to a more sophisticated, piecewise linear potential.

For our further investigations, it will be useful to have a handy expression for the critical supply of energy, $d_{2}^{c r i t}$, which allows a stable "uphill" motion. This will be derived in the following, with only a few approximations. For the parameters used during the computer simulations discussed later, Fig. 4 and Table I indicate that the square root in Eq. (24) is imaginary, thus the stability of the 
Frank Schweitzer, Benno Tilch, Werner Ebeling:

Uphill Motion of Active Brownian Particles in Piecewise Linear Potentials

European Journal of Physics B (1999, in press)
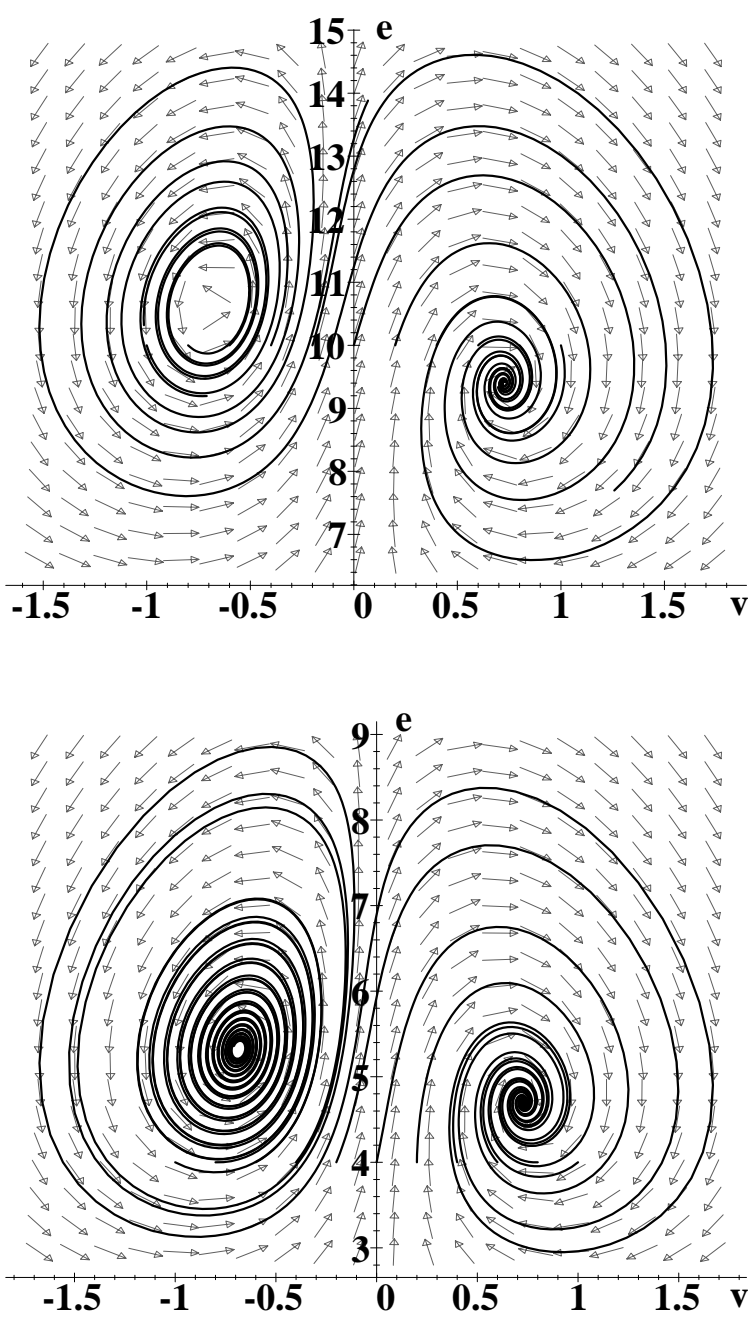

Figure 3: Phase trajectories in the $\{v, e\}$ phase space for the motion into or against the direction of the driving force, which correspond either to positive or negative velocities. (a: top) $d_{2}=2.0$ corresponding to an instable "uphill" motion, (b: bottom) $d_{2}=4.0$ corresponding to a stable "uphill" motion, respectively. Other parameters see Fig. 1.

solutions depends on the condition:

$$
\gamma_{0}+c+d_{2}\left(v_{0}^{2}-e_{0}\right) \geq 0
$$

If we insert the stationary value $e_{0}$, Eq. (16), Eq. (25) leads to a 4 th order inequation for $v_{0}$ to 


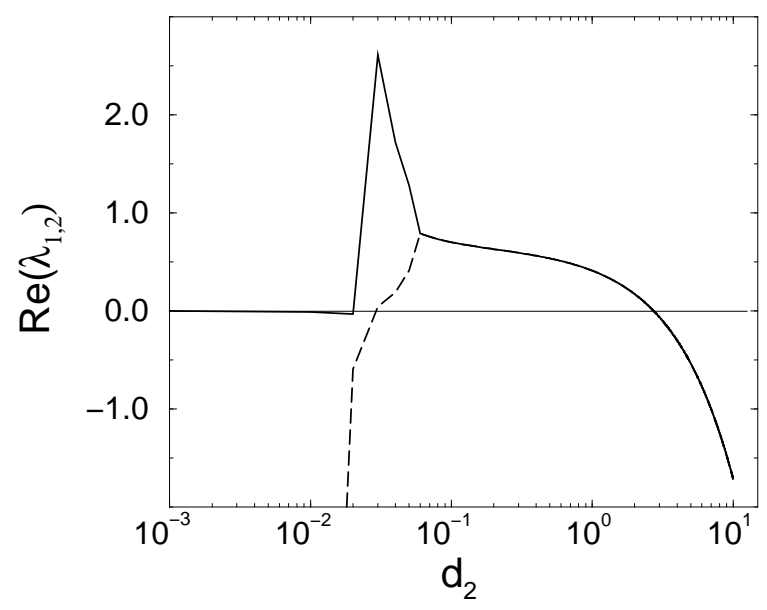

Figure 4: Real part of $\lambda^{(1,2)}$, Eq. (24), vs. conversion parameter $d_{2}$ for the stationary motion against the force, $\boldsymbol{F}$. Parameters see Fig. 1.

obtain stability:

$$
\left(\gamma_{0} c-d_{2} q_{0}\right) \leq v_{0}^{4} d_{2}^{2}+v_{0}^{2}\left(\gamma_{0} d_{2}+2 c d_{2}\right)+c^{2}
$$

For a stable stationary motion of the particle, both Eq. (17) and Eq. (26) have to be satisfied.

The critical condition for stability just results from the equality in Eq. (26), which then provides a replacement for the prefactor $\left(\gamma_{0} c-d_{2} q_{0}\right)$ in Eq. (17). If we insert the critical condition into Eq. (17), we arrive at a 5 th order equation for $v_{0}$ :

$$
\boldsymbol{v}_{0}^{5}+\boldsymbol{v}_{0}^{3}\left(\frac{2 c}{d_{2}}\right)+v_{0}^{2}\left(\frac{\boldsymbol{F}}{d_{2}}\right)+\boldsymbol{v}_{0}\left(\frac{c}{d_{2}}\right)^{2}+\frac{c \boldsymbol{F}}{d_{2}^{2}}=0
$$

In order to simplify the further discussion, we assume that the internal dissipation is negligible, $c=0$. Then, Eq. (27) gives the simple nontrivial solution:

$$
\boldsymbol{v}_{0}^{3}=-\frac{\boldsymbol{F}}{d_{2}} ; \quad \text { if } \quad c=0
$$

This expression can be used to eliminate the stationary velocity, $\boldsymbol{v}_{0}$, in Eq. (26). With the assumption $c=0$, we obtain now from the critical condition, i.e. from the equality in Eq. (26), a relation between the force, $\boldsymbol{F}$, and the conversion parameter, $d_{2}$. Combining Eq. (26) and Eq. (28) results in

$$
(-F)^{4 / 3} d_{2}+\gamma_{0}(-F)^{2 / 3} d_{2}^{2 / 3}-q_{0} d_{2}^{4 / 3}=0
$$


Because of $d_{2}>0$, the trivial and the negative solution of Eq. (29) can be neglected, and we finally arrive at the following critical relation for $d_{2}(F)$ :

$$
d_{2}^{c r i t}=\frac{F^{4}}{8 q_{0}^{3}}\left(1+\sqrt{1+\frac{4 \gamma_{0} q_{0}}{F^{2}}}\right)^{3}
$$

Fig. 5 shows $d_{2}^{\text {crit }}$ as a function of the force, $F$. In the limit of negligible internal dissipation, the relation $d_{2}^{\text {crit }}(F)$ describes how much power has to be supplied by the internal energy depot in order to allow a stable motion of the particle in both directions, in particular a stable uphill motion of the particle.

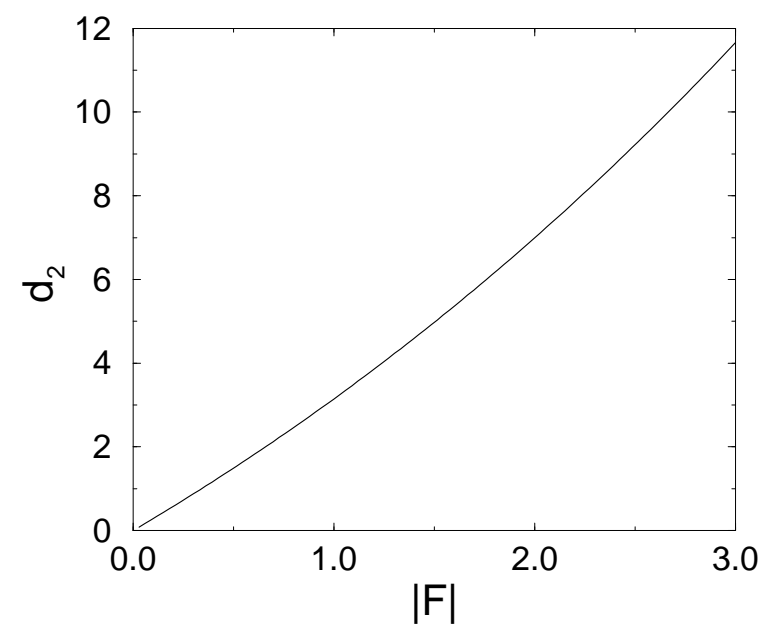

Figure 5: Critical conversion rate, $d_{2}^{\text {crit }}$, Eq. (30), vs. amount of the driving force, $|F|$, to allow a stable motion of the particle both "downhill" and "uphill" (cf. Fig. 2). For $d_{2}>d_{2}^{\text {crit }}$, the particle is able to move also against the direction of the force. Parameters see Fig. 1. 


\section{Deterministic Motion in a Ratchet Potential}

\subsection{Simulation Results for a Single Pumped Particle}

For further investigations of the motion of pumped particles, we specify the potential $U(\boldsymbol{x})$ as a piecewise linear, asymmetric potential (cf. Fig. 6), which is known as a ratchet potential:

$$
U(x)=\left\{\begin{array}{r}
\frac{U_{0}}{b}\{x-n L\} \\
\text { if } n L \leq x \leq n L+b \\
\frac{U_{0}}{L-b}\{(n+1) L-x\} \\
\text { if } n L+b \leq x \leq(n+1) L \\
\quad(n=0,1,2, \ldots)
\end{array}\right.
$$

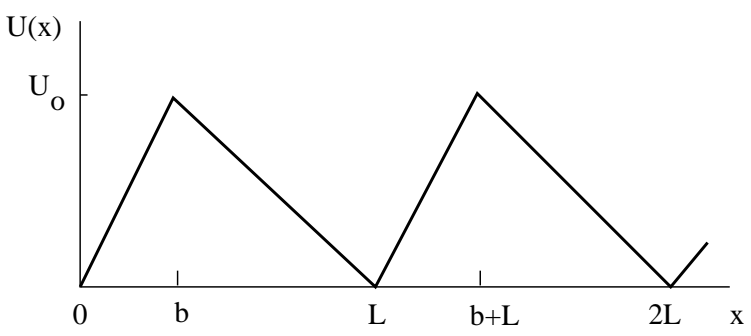

Figure 6: Sketch of the asymmetric potential $U(x)$ (eq. (31)). For the computer simulations, the following values are used: $\mathrm{b}=4, \mathrm{~L}=12, U_{0}=7$ in arbitrary units.

Further, we will use the following abbreviations with respect to the potential $U(x)$, Eq. (31). The index $i=\{1,2\}$ refers to the two pieces of the potential, $l_{1}=b, l_{2}=L-b$. The asymmetry parameter $a$ should describe the ratio of the two pieces, and $\boldsymbol{F}=-\boldsymbol{\nabla} U=$ const. is the force resulting from the gradient of the piecewise linear potential. Hence, for the potential $U(x)$, Eq. (31), the following relations yield:

$$
\begin{aligned}
& F_{1}=-\frac{U_{0}}{b} ; \quad F_{2}=\frac{U_{0}}{L-b} ; \quad a=\frac{l_{2}}{l_{1}}=\frac{L-b}{b}=-\frac{F_{1}}{F_{2}} \\
& F_{1}=-\frac{U_{0}}{L}(1+a) ; \quad F_{2}=\frac{U_{0}}{L} \frac{1+a}{a}
\end{aligned}
$$

Whether or not the particle will be able to leave one of the potential wells described by Eq. (31), depends in a first approximation on the height of the potential barrier, $U_{0}$, and on the kinetic energy of the particle. For particles with internal energy depot, the actual velocity depends also on the conversion of internal into kinetic energy, Eq. (7). In agreement with the investigations in the previous section, we can in principle distinguish between two different types of motion: (i) a bound motion, i.e. the particle will not leave the potential well because of the subcritical supply 
of energy from the depot, but its position might oscillate within the boundaries, (ii) an unbound motion, i.e. the particle will be able to leave the potential well because of the supercritical supply of energy and move freely. Both types of motion have analogies to the localized and delocalized states of electrons in solid state physics as will be discussed in more detail in a forthcoming paper. In the following, we discuss computer simulations of the deterministic motion of one pumped Brownian particle in a ratchet potential. The particle (mass $m=1$ ) starts its motion outside the potential extrema; hence, there is an initial force on the particle. The results for a single particle are shown in Fig. 7, where two different sets of parameters are used:

(i) a small internal dissipation, $c$, which means a nearly ideal energy depot, and a large friction coefficient, $\gamma_{0}$, resulting in a strongly damped motion,

(ii) an internal dissipation, $c$, 10 times larger, an energy influx, $q_{0}$, ten times smaller, and a friction coefficient, $\gamma_{0}, 100$ times smaller than in (i), resulting in a weakly damped motion,

We note, that in the computer simulations always the complete set of Eq. (7) for the particles is solved, regardless of the possible approximations. The trajectories $\boldsymbol{x}(t)$ in Fig. 7 indicate a nearly uniform motion of the particle into one direction. The continuous motion corresponds to a delocalized state of the particle in the ratchet potential, whereas for subcritical energetic conditions only localized states exist. The transition from localized to delocalized states will be discussed in more detail in a forthcoming paper [31].

As shown in Fig. 7a, the less damped motion of a single particle may result in steady oscillations in the velocity, $\boldsymbol{v}(t)$, and the energy depot, $e(t)$. Only if the damping is large enough, the velocity and the energy depot may reach constant values (cf. Fig. 7b). These values are of course different for each piece of the potential, hence the periodical movement through every maximum or minimum of the potential results in jumps both in the velocity and the energy depot, which are followed by oscillations. In the phase space shown in Fig. 8a, the motion of the particle appears as a transition between two stable fix points, each of which describes the stable motion on one flank.

In the following, we restrict the discussion to the strongly damped case. The oscillations which occur in $v$ and $e$ are damped out on a characteristic time scale, $\tau=1 / \gamma_{0}$. If we assume that the particle moves on the different pieces of the potential $\{b, L-b\}$ (cf. Fig. 6) during the two characteristic time intervals: $T_{b}=b / v_{b}$ and $T_{L-b}=(L-b) / v_{(L-b)}$, then the particle is subject to a constant force only as long as $\tau \ll T_{b}$ or $\tau \ll T_{L-b}$, respectively. For times larger than the characteristic time, $\tau$, the motion of the particle can be described by the equation of the overdamped limit, Eq. (11). If we neglect again stochastic influences, Eq. (11) can be rewritten in the form:

$$
0=-\left[\gamma_{0}-d_{2} e_{0}\right] \boldsymbol{v}_{0}+\boldsymbol{F}
$$

where $|\dot{\boldsymbol{x}}|=\left|\boldsymbol{v}_{0}\right|=$ const. is the velocity in the overdamped limit and $\boldsymbol{F}=\left\{F_{1}, F_{2}\right\}$ is defined by Eq. (32). The stationary value for the internal energy depot, $e_{0}$, is given by Eq. (16). If we assume 
Frank Schweitzer, Benno Tilch, Werner Ebeling:

Uphill Motion of Active Brownian Particles in Piecewise Linear Potentials

European Journal of Physics B (1999, in press)
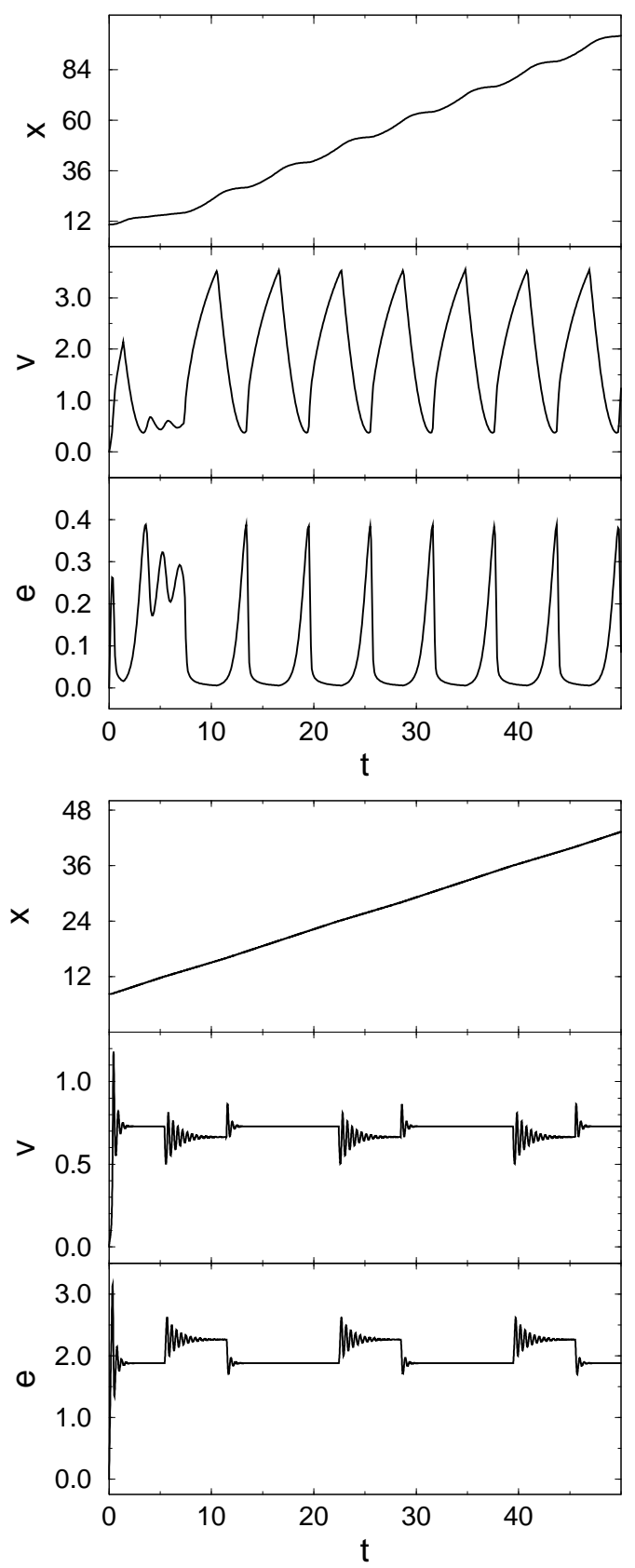

Figure 7: Trajectory $x(t)$, velocity $v(t)$ and energy depot $e(t)$ for a single particle moving in a ratchet potential (Fig. 6). Parameters: (a: top) $q_{0}=1.0, \gamma=0.2, c=0.1, d_{2}=14.0$, (b: bottom) $q_{0}=10, \gamma_{0}=20, c=0.01, d_{2}=10$. Initial conditions: $x(0) \in[4,12], v(0)=0, e(0)=0$ 
Frank Schweitzer, Benno Tilch, Werner Ebeling:

Uphill Motion of Active Brownian Particles in Piecewise Linear Potentials

European Journal of Physics B (1999, in press)
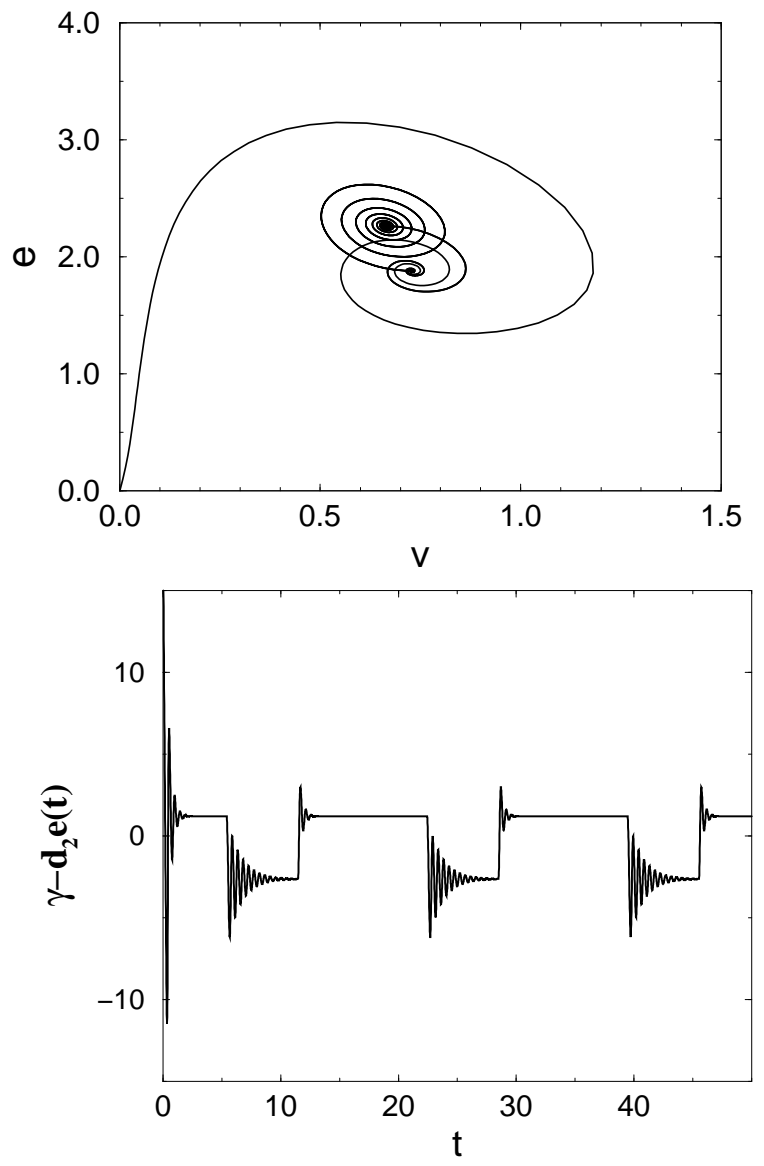

Figure 8: (a: top) Energy depot $e(t)$ vs. velocity $v(t)$, (b: bottom) prefactor: $\gamma-d_{2} e(t)$ for the strongly damped motion of a single particle (data from Fig. $7 \mathrm{~b}$ ).

again that the term $c \boldsymbol{F}$ is small, then the constant velocity can be calculated from Eq. (20), with $\boldsymbol{F}$ specified as $F_{1}$ or $F_{2}$, respectively. For any constant force $F$, there are two possible non-trivial solutions of Eq. (20): a positive and a negative velocity with different, but constant amount, which depend on the gradient of the potential. The nontrivial values $v_{0} \neq 0$ of Eq. (20) can be compared with the constant values obtained in the simulations, and we find:

Fig. $7 \mathrm{~b} \quad$ Eq. (20)

$\begin{array}{ccc}\text { lower value } v_{0} & 0.665 & 0.6642 \\ \text { upper value } v_{0} & 0.728 & 0.7288\end{array}$

The eqs. (11), (33) for the overdamped limit indicate that the dynamics remarkably depends on the sign of the prefactor $\gamma_{0}-d_{2} e_{0}$, which governs the influence of the potential and the stochastic force. 
Therefore, the prefactor should be discussed in more detail, now. Fig. 8b shows that the prefactor $\gamma_{0}-d_{2} e(t)$ displays a behavior similar to the velocity, Fig. $7 \mathrm{~b}$. The prefactor jumps between a positive and a negative constant value, which can be approximated by means of the constant, $e_{0}$, Eq. (16), reached after the oscillations damped out. It is shown that the jump occurs at the same time when the gradient of the potential changes its sign. This can be also proved analytically. Using Eqs. (10), (20), the prefactor $\gamma_{0}-d_{2} e_{0}$ in Eq. (11) and Eq. (33), respectively, can be rewritten and we find after a short calculation:

$$
\frac{1}{\gamma_{0}-d_{2} e_{0}}=\frac{1}{2 \gamma_{0} F_{i}}\left[F_{i} \pm \sqrt{F_{i}^{2}+4\left(q_{0} \gamma_{0}-c \gamma_{0}^{2} / d_{2}\right)}\right]
$$

This means that the product of the prefactor and the potential gradient always has the same positive (or negative) sign, and the direction of motion for the particle is only determined by the initial condition.

The prefactor $\gamma_{0}-d_{2} e_{0}$ describes the balance between dissipation and the energy supply from the internal depot of the particle. Therefore it is expected that the time average of the prefactor should be zero, when averaged over one time period $T$ :

$$
\left\langle\gamma_{0}-d_{2} e_{0}\right\rangle T=0
$$

This can be proven using $T=T_{b}+T_{L-b}$ as discussed above. The values $v_{b}, v_{L-b}$ and $e_{b}, e_{L-b}$ for the related velocity and energy depot along the different pieces of the potential can be calculated by means of Eqs. (20), (33) which leads directly to Eq. (36).

\subsection{Investigation of the Net Current for an Ensemble of Particles}

Let us now discuss the deterministic motion of an ensemble of $N$ pumped Brownian particles in a ratchet potential. For the computer simulations, we have assumed that the start locations of the particles are equally distributed over the first period of the potential, $\{0, L\}$ and their initial velocity is zero. In the deterministic case, the direction of motion and the velocity at any time $t$ are mainly determined by the initial conditions. Hence, particles with an initial position between $\{0, b\}$, which initially feel a force into the negative direction, most likely move with a negative velocity, whereas particles with an initial position between $\{b, L\}$ most likely move into the positive direction. This is also shown in Fig. 9a, where the velocity $v$ is plotted versus the initial position of the particles. Oscillations occur only at the minima and maxima of the related potential, indicating a strong sensitivity to the initial condition in these regions. The distribution of the final velocity is shown in Fig. 9b.

From Fig. 9b we see two main currents of particles occuring, one with a positive and one with a negative velocity, which can be approximated by Eq. (20). The net current, however, has a positive 
Frank Schweitzer, Benno Tilch, Werner Ebeling:

Uphill Motion of Active Brownian Particles in Piecewise Linear Potentials

European Journal of Physics B (1999, in press)
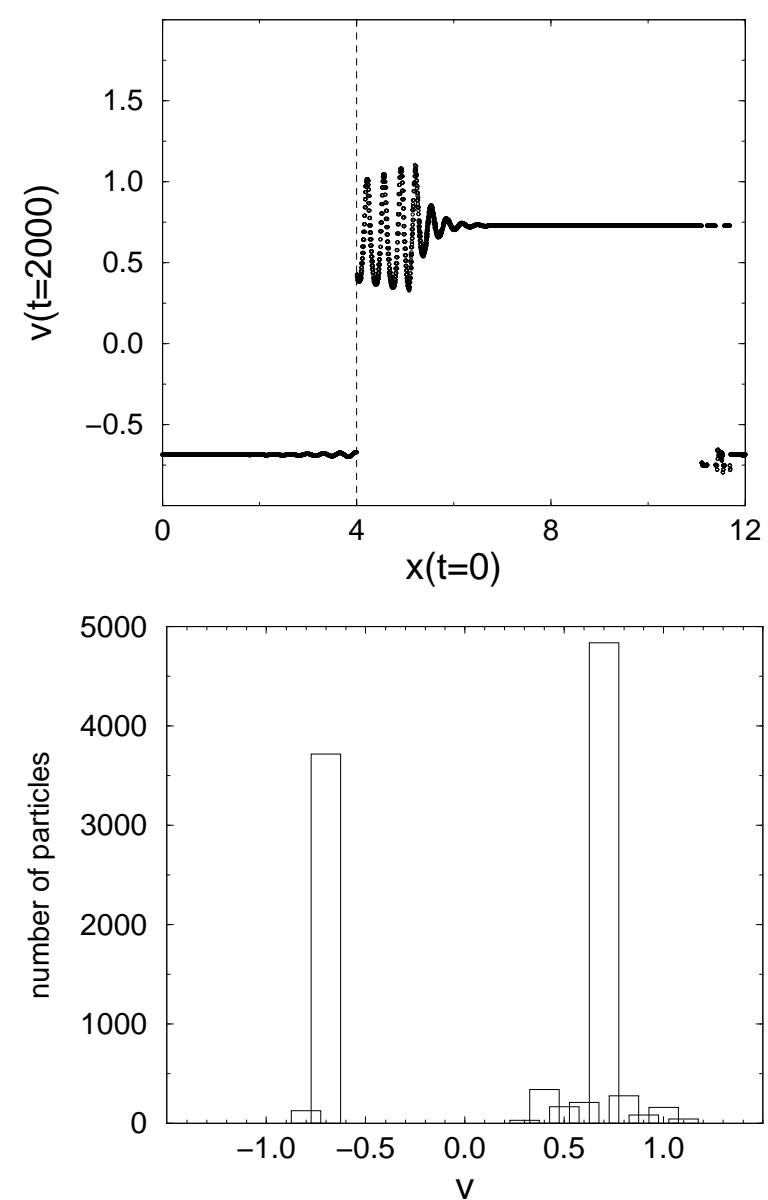

Figure 9: (a: top) Final velocity $v_{e}$ after $\mathrm{t}=2.000$ simulation steps (averaged for 10.000 particles) vs. initial location $x_{0}$ of the particles. (b: bottom) Distribution of the final velocity $v_{e}$. Parameters see Fig. 7b, initial locations of the particles are equally distributed over the first period of the ratchet potential $\{0, L\}$.

direction, since most of the particles start with the matching initial condition. The time dependence of the averages is shown in Fig. 10. The long-term oscillations in the average velocity and the average energy depot result from the superposition of the velocities, which are sharply peaked around the two dominating values (cf. Fig. 9b).

The existence of periodic stationary solutions, $\boldsymbol{v}_{0}(x)=\boldsymbol{v}_{0}(x \pm L)$, requires that the particles are able to escape from the initial period of the potential. For a continuous motion which corresponds to delocalized states, the particles must be able to move "uphill" on one or both flanks of the ratchet potential. In Sect. 3, we already investigated the necessary conditions for such a motion for a single flank, and found a critical condition for the conversion rate, $d_{2}$, Eq. (30). In order to demonstrate 
Frank Schweitzer, Benno Tilch, Werner Ebeling:

Uphill Motion of Active Brownian Particles in Piecewise Linear Potentials

European Journal of Physics B (1999, in press)

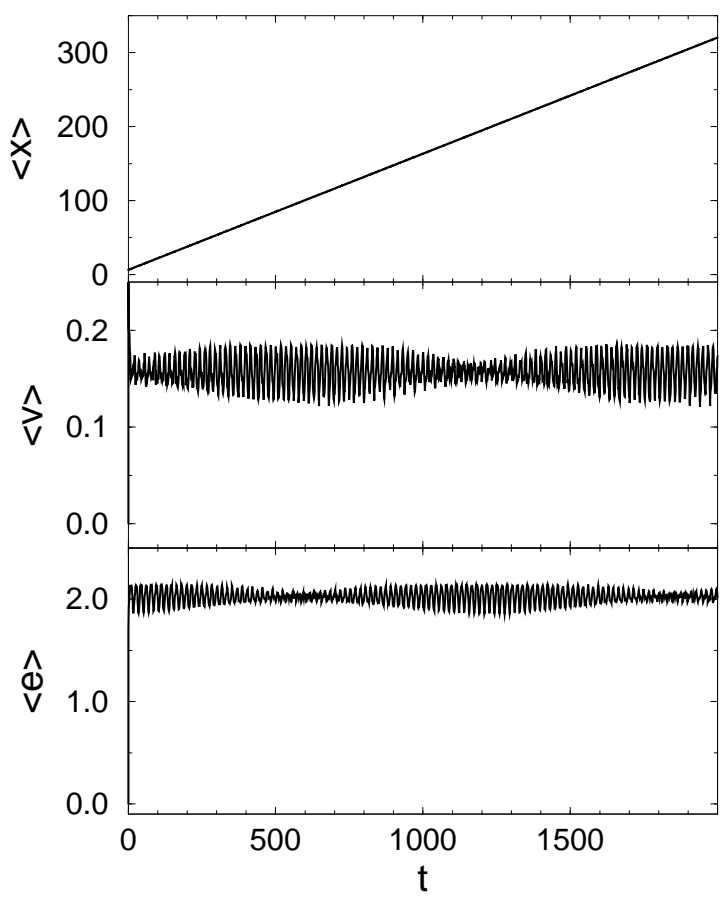

Figure 10: Averaged location $\langle x\rangle$, velocity $\langle v\rangle$ and energy depot $\langle e\rangle$ of 10.000 particles vs. time $t$. Parameters see Fig. $7 \mathrm{~b}$.

the applicability of Eq. (30) for the ratchet potential, we have investigated the dependence of the net current, expressed by the mean velocity $\langle v\rangle$, on the conversion rate, $d_{2}$, for the overdamped case. The results of computer simulations are shown in Fig. 11.

In Fig. 11, we see the existence of two different critical values for the parameter $d_{2}$, which correspond to the onset of a negative net current at $d_{2}^{\text {crit } 1}$ and a positive net current at $d_{2}^{\text {crit2 }}$. For values of $d_{2}$ near zero and less than $d_{2}^{\text {crit } 1}$, there is no net current at all. This is due to the subcritical supply of energy from the internal depot, which does not allow an uphill motion on any flank of the potential. Consequently, after the initial downhill motion, all particles come to rest in the minima of the ratchet potential, with $v_{0}=0$ as the only stationary solution for the velocity. With an increasing value of $d_{2}$, we see the occurence of a negative net current at $d_{2}^{c r i t 1}$. That means, the energy depot provides enough energy for the uphill motion along the flank with the lower slope, which, in our example, is the one with $F=7 / 8$ (cf. Fig. 6). If we insert this value for $F$ into the critical condition, Eq. (30), a value $d_{2}^{\text {crit } 1}=2.715$ is obtained, which agrees with the onset of the negative current in the computer simulations, Fig. 11. 


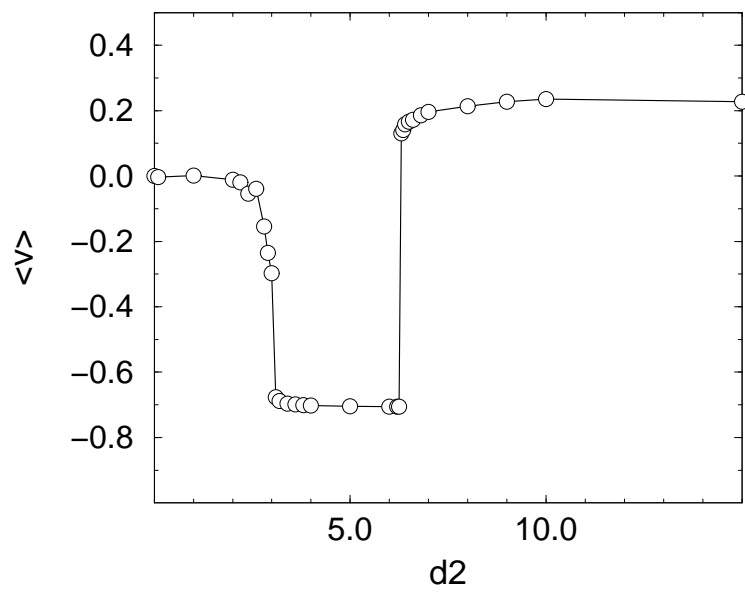

Figure 11: Average velocity $\langle v\rangle$ vs. conversion parameter $d_{2}$. The data points are obtained from simulations of 10.000 particles with arbitrary initial positions in the first period of the ratchet potential. Other parameters see Fig. 7b.

For $d_{2}^{\text {crit } 1} \leq d_{2} \leq d_{2}^{\text {crit } 2}$, a stable motion of the particles up and down the flank with the lower slope is possible, but the same does not necessarily apply for the steeper slope. Hence, particles which start on the lower slope with a positive velocity, cannot continue their motion into the positive direction since they are not able to climb up the steeper slope. Consequently, they turn their direction on the steeper slope, then move downhill driven by the force into the negative direction, and continue to move into the negative direction while climbing up the lower slope. Therefore, for values of the conversion rate between $d_{2}^{c r i t 1}$ and $d_{2}^{\text {crit } 2}$, we only have an unimodal distribution of the velocity centered around the negative value:

$$
v_{1}=\frac{F}{2 \gamma_{0}}-\sqrt{\frac{F^{2}}{4 \gamma_{0}^{2}}+\frac{q_{0}}{\gamma_{0}}}=-0.6855 \text { for } F=\frac{7}{8}
$$

which is independent of $d_{2}$ if the term $(c / d 2)$ in Eq. (20) is negligible, which holds for the considered case. For $d_{2}>d_{2}^{\text {crit } 2}$, the energy depot also supplies enough energy for the particles to climb up the steeper slope, consequently a periodic motion of the particles into the positive direction becomes possible, now. In our example, the steeper slope corresponds to the force $F=-7 / 4$ (cf. Fig. 6) which yields a critical value $d_{2}^{\text {crit } 1}=5.985$, obtained by means of Eq. (30). This result agrees with the onset of the positive current in the computer simulations, Fig. 11.

For $d_{2}>d_{2}^{c r i t 2}$, we have a bimodal velocity distribution, as also shown in Fig. 9b. The net current, which results from the average of the two main currents, has a positive direction in the deterministic case, because most of the particles start into a positive direction, as discussed above. We may simply assume, that the number of particles in each direction is roughly proportional to the length of the 
flank where they started from, which is also indicated by the velocity distribution, Fig. 9b. Then the mean velocity in the strongly damped case can be approximated by:

$$
\langle v\rangle=\frac{1}{N} \sum_{i=1}^{N} v_{i}=\frac{1}{3} v_{1}+\frac{2}{3} v_{2}
$$

where $v_{1}$ and $v_{2}$ are the stationary velocities on each flank, which, in the limit of an nearly ideal energy depot, can be determined from Eq. (20). With the negative velocity, $v_{1}$, Eq. (37), and the positive velocity,

$$
v_{2}=\frac{F}{2 \gamma_{0}}+\sqrt{\frac{F^{2}}{4 \gamma_{0}^{2}}+\frac{q_{0}}{\gamma_{0}}}=0.664 \text { for } F=-\frac{7}{4}
$$

we find from Eq. (38) for $d_{2}>d_{2}^{\text {crit2 }}$ an average velocity, $\langle v\rangle=0.216$, which also agrees with the computer simulations, Fig. 11.

The results of the computer simulations have demonstrated that in the deterministic case for the given special initial condition, i.e. the equal distribution of particles over the ratchet period, the direction of the net current can be adjusted by choosing the appropriate values of the conversion rate, $d_{2}$.

The critical values for $d_{2}$, on the other hand, depend on the slope of the two flanks of the potential, expressed by the force $F$. Lower slopes also correspond to lower values of the conversion rate, because less power is needed for the uphill motion.

We conclude our results by investigating the influence of the slope on the establishment of a positive or negative net current. With a fixed height of the potential barrier, $U_{0}$, and a fixed length $L$, the ratio of the two different slopes is described by the asymmetry parameter $a=l_{2} / l_{1}=-F_{1} / F_{2}$, Eq. (32). The occurence of a current in the ratchet potential requires the possibility of uphill-motion, which depends on the critical supply of energy, described by Eq. (30). In order to obtain the critical value for the asymmetry of the potential, we replace the force $F$ in Eq. (30) by the parameter $a$, Eq. (32). In our example, the flank $l_{1}$ of the potential has the steeper slope, so the critical condition is determined by $F_{1}=U_{0} / L(1+a)$. As the result, we find:

$$
a^{\text {crit }}=\frac{L}{U_{0}}\left[-\frac{\gamma_{0}}{2} d_{2}^{-1 / 3}+\sqrt{\frac{\gamma^{2}}{4} d_{2}^{-2 / 3}+q_{0} d_{2}^{1 / 3}}\right]^{3 / 2}-1
$$

$a^{\text {crit }} \geq 1$ gives the critical value for the asymmetry, which may result in a reversal of the net current. For $a>a^{\text {crit }}$, the flank $l_{1}$ is too steep for the particles, therefore only a negative current can occur which corresponds to the unimodal velocity distribution discussed above. For $1<a<a^{\text {crit }}$, however, the particles are able to move uphill either flank. Hence, also a positive current can establish and the velocity distribution becomes bimodal, which results in a positive net current. 


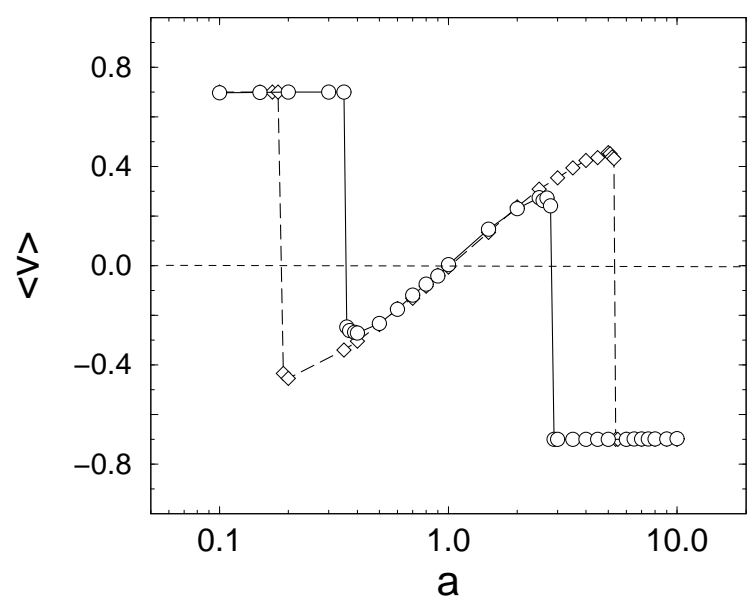

Figure 12: Average velocity $\langle v\rangle$ vs. asymmetry parameter $a$, Eq. (32). The data points are obtained from simulations of 10.000 particles with arbitrary initial positions in the first period of the ratchet potential. $(\circ): d_{2}=10(-),(\diamond): d_{2}=20(--)$. Other parameters see Fig. $7 \mathrm{~b}$.

The current reversal from a negative to a positive net current is shown in Fig. 12. Dependent on the value of the conversion rate, $d_{2}$, we see the switch from the negative to the positive value of the net current at a critical value of the asymmetry parameter $a$. Because of the definition of $a$, the results for $a<1$, are the inverse of the results for $a>1$. Obviously, for a symmetric ratchet, $a=1$ no net current occurs, because the two main currents compensate. From Eq. (40), we obtain $a^{\text {crit }}=3.5$ for $d_{2}=10$, and $a^{\text {crit }}=6.5$ for $d_{2}=20$, which both agree with the results of the computer simulations, Fig. 12. Further, the results of Fig. 12 shows that the stationary velocities are independent of $d_{2}$ in the limit of an nearly ideal energy depot, which is also indicated by Eq. (20).

\section{Summary and Conclusions}

In this paper, we have investigated the dynamics of Brownian particles with an internal energy depot, which can be filled by the take-up of energy from the environment. This extension which is inspired by the biological features of active motion, has several aspects: (i) the spatial or spatialtemporal inhomogeneous distribution of energy from the environment can be considered, (ii) the supply of depot energy for different activities, i.e. for accelerated motion or signal-response behavior, can be modeled, (iii) in addition to external dissipative processes caused by friction, internal dissipation can be included, (iv) the adjustment of the time scale for the relaxation of the energy depot allows to consider delay effects, i.e. for the acceleration of motion.

In this paper, we are mainly interested in the question how the internal energy depot will affect the particle's motion in an external potential. We found that provided some supercritical energetic 
conditions, the Brownian particles are able to move in a "high velocity" or active mode of motion characterized by a velocity much larger than the Stokes velocity. The latter one can be denoted as the passive mode of motion. In addition to stochastic forces, it is basically determined by the response to the gradient of an external potential. One of the possible active modes of motion can be understood as a continuation of the passive or Stokes mode, the particles motion in the direction of the force being accelerated.

Additionally, we also found an active mode which describes a motion of the particle against the gradient of an external potential. We have investigated the critical conditions for the deterministic motion of the particle in the overdamped limit for the case of a linear potential. We found that the "uphill" motion is described by two critical conversion rates. The bifurcation diagram shows that, at a critical value $d_{2}^{b i f}$, the possibility of an "uphill" motion appears as a new solution for the stationary velocity, i.e. "uphill" motion requires the existence of multiple stationary solution for the velocity. However, this active mode remains unstable as long as the conversion rate is below a second critical value $d_{2}^{\text {crit }}$, Eq. (30), which depends on parameters describing the energy balance, such as the friction coefficient, $\gamma_{0}$, and the influx of energy into the depot, $q_{0}$, and on the gradient of the potential, i.e. the resulting force $\boldsymbol{F}$. A stable "uphill" motion occurs only for a supercritical conversion rate.

These results allow us to interpret the deterministic motion of an ensemble of particles in a ratchet potential, i.e. a piecewise linear, periodic asymmetric potential with two different slopes. Initially, the particles are equally distributed over the first period of the potential. In order to produce a current, the particles must be able to escape from the potential well, which depends on the supply of energy. We are able to find two different critical conversion rates, which describe the onset of a directed current into the different directions. As long as the particles are only able to move uphill the flank with the lower slope, we have an unimodal velocity distribution, corresponding to a negative net current. But if the particles are able to move uphill either flank, we find a bimodal velocity distribution, and a positive net current for the given conditions. Hence, in the deterministic case the direction of the net current can be controlled via a single parameter, $d_{2}$, which describes the conversion of internal into kinetic energy. We are able to calculate the critical parameter, as well as the resulting velocity of the net current from analytical considerations, which agree with the results found in the computer simulations. Alternatively, the critical supply of energy can be also reformulated in terms of a critical asymmetry of the ratchet potential, which restricts the occurence of a (positive or negative) net current.

To conclude the results obtained, we have shown that an ensemble of pumped Brownian particles moving in a ratchet potential can produce a directed net current. In this respect, our result agrees with the conclusions of other physical ratchet models which have been proposed to reveal the microscopic mechanisms resulting in directed movement. Due to [19, p. 295] a ratchet system which is meant to be "a system that is able to transport particles in a periodic structure with nonzero macroscopic velocity although on average no macroscopic force is acting." Indeed, we have shown 
in Eq. (36) that for the stationary approximation $\left\langle\gamma_{0}-d_{2} e_{0}\right\rangle \tau=0$ holds, i.e. the force acting on the particle is of zero average with respect to one period, $\tau$. However, different from other ratchet models, ie. the rocking ratchet $[27,28,30]$ or the diffusion ratchet [29] which assume a spatially uniform time-periodic force, the force in our model switches between two constant values, dependent on the moving direction and the flank the particle is moving on. Hence, it does not represent a spatially uniform force, and we may conclude that the mechanism of motion which Eq. (11) is based on, should be different from the previous mechanisms which originate directed motion in a ratchet potential.

In this paper, we have restricted the discussion to the deterministic motion of Brownian particles with an internal energy depot. We note that the stochastic motion of an ensemble of Brownian particles in a ratchet potential will be investigated in detail in a forthcoming paper [31], which pays particular attention to the influence of stochastic effects on the establishment of the net current. Additionally, we will also discuss the non-equilibrium distribution functions for Brownian particles with internal energy depot [26].

As a final remark, we note in a more general sense that the introduction of an internal energy depot adds an interesting and new element to the known model of Brownian particles, which can be useful in two different respects. With respect to the physical aspects, the dynamical system now has a new degree of freedom, which increases the phase space, $\Gamma=\left\{x_{i}, v_{i}, e\right\}$. Due to the additional energy supply, the system is driven into non-equilibrium. Hence, a qualitative new behavior in the particle's motion can be obtained.

With respect to possible biological aspects, the extension of the Brownian particle model by mechanisms of energy take-up, storage and conversion, should contribute to the development of a $m i$ croscopic theory of active biological motion. The final goal of such a project could be a microscopic image of well known phenomenological models of biological motion, taking into account energy balances that are related to the mechanisms of energy pumping and energy dissipation.

\section{References}

[1] A. S. Mikhailov and D. Meinköhn, Self-Motion in Physico-Chemical Systems Far from Thermal Equilibrium in Stochastic Dynamics, edited by L. Schimansky-Geier and T. Pöschel (Springer, Berlin, 1997).

[2] T. Vicsek, A. Czirok, E. Ben-Jacob, I. Cohen and O. Shochet, Novel Type of Phase Transition in a System of Self-Driven Particles, Phys. Rev. Lett. 75 (1995) 1226.

[3] E. V. Albano, Self-organized collective displacements of self-driven individuals Phys. Rev. Lett. 77 (1996) 2129. 
Frank Schweitzer, Benno Tilch, Werner Ebeling:

Uphill Motion of Active Brownian Particles in Piecewise Linear Potentials

European Journal of Physics B (1999, in press)

[4] D. Helbing and T. Vicsek, Optimal Self-Organization, New Journal of Physics 1 (1999) 13.1 (http://www.njp.org/).

[5] R.T. Tranquillo and D. Lauffenburger, Stochastic models of leukocyte chemosensory movement, J. Mathematical Biology 25 (1987) 229.

[6] H.G. Othmer, S.R. Dunbar and W. Alt, Models of dispersal in biological systems, J. Mathematical Biology 26 (1988) 263.

[7] Biological Motion, edited by W. Alt and G. Hoffmann (Springer, Berlin, 1990).

[8] R. Dickinson and R.T. Tranquillo, A stochastic model for adhesion-mediated cell random motility and haptotaxis, J. Mathematical Biology 31 (1993) 563.

[9] M. Schienbein and H. Gruler, Langevin Equation, Fokker-Planck Equation and Cell Migration, Bull. Mathem. Biology 55 (1993) 585.

[10] F. Schweitzer and L. Schimansky-Geier, Clustering of Active Walkers in a Two-Component System, Physica A 206 (1994) 359.

[11] O. Steuernagel, W. Ebeling and V. Calenbuhr, An Elementary Model for Directed Active Motion, Chaos, Solitons \& Fractals 4 (1994) 1917.

[12] L. Schimansky-Geier, M. Mieth, H. Rose and H. Malchow, Structure Formation by Active Brownian Particles, Physics Lett. A 207 (1995) 140.

[13] F. Schweitzer, Active Brownian Particles: Artificial Agents in Physics, in Stochastic Dynamics, edited by L. Schimansky-Geier and T. Pöschel (Springer, Berlin, 1997).

[14] F. Schweitzer, W. Ebeling and B. Tilch, Complex Motion of Brownian Particles with Energy Depots, Phys. Rev. Lett. 80 (1998) 5044.

[15] W. Ebeling, F. Schweitzer and B. Tilch, Active Brownian Particles with Energy Depots Modelling Animal Mobility, BioSystems 49 (1999) 17.

[16] M.O. Magnasco, Molecular Combustion Motors, Phys. Rev. Lett. 72 (1994) 2656.

[17] R.D. Astumian and M. Bier, Fluctuation Driven Ratchets: Molecular Motors, Phys. Rev. Lett. 72 (1994) 1766.

[18] F. Jülicher and J. Prost, Phys. Rev. Lett. 75 (1995) 2618.

[19] P. Hänggi and R. Bartussek, Brownian Rectifiers: How to Convert Brownian Motion into Directed Transport, in Nonlinear Physics of Complex Systems - Current Status and Future Trends, edited by J. Parisi, S.C. Müller and W. Zimmermann (Springer, Berlin, 1996). 
[20] J. Maddox, Directed motion from random noise, Nature 369 (1994) 181.

[21] M. O. Magnasco, Forced Thermal Ratchets, Phys. Rev. Lett. 71 (1993) 1477.

[22] J. Luczka, R. Bartussek and P. Hänggi, White Noise Induced Transport in Periodic Structures, Europhys. Lett. 31 (1995) 431.

[23] M. M. Millonas and M. I. Dykman, Transport and current reversal in stochastically driven ratchets, Physics Lett. A 185 (1994) 65.

[24] J. Rousselet, L. Salome, A. Ajdari and J. Prost, Directional motion of brownian particles induced by a periodic asymmetric potential, Nature 370 (1994) 446.

[25] U. Zürcher and Ch. R. Doering, Thermally activated escape over fluctuating barriers, Phys. Rev. E 47 (1993) 3862.

[26] U. Erdmann, W. Ebeling, L. Schimansky-Geier and F. Schweitzer, Brownian particles far from equilibrium, Europ. J. Physics B (1999, in press) .

[27] R. Bartussek, P. Hänggi and J.G. Kissner, Europhysics Letters 28 (1994) 459.

[28] B. Lindner, L. Schimansky-Geier, P. Reimann and P. Hänggi, Mass Separation by Ratchets, Proc. American Phys. Soc. 411 (1997) 309.

[29] P. Reimann, R. Bartussek, R. Häußler and P. Hänggi, Physics Letters A 215 (1996) 26.

[30] P. S. Landa, Noise-induced transport of Brownian particles with consideration for their mass, Phys. Rev. E 58 (1998) 1325.

[31] B. Tilch, F. Schweitzer and W. Ebeling, Directed Motion of Brownian Particles With Internal Energy Depot, Physica A 273 (1999) 294-314. 